\title{
ON STRUCTURE AND TKK ALGEBRAS FOR JORDAN SUPERALGEBRAS
}

\author{
SIGISWALD BARBIER AND KEVIN COULEMBIER
}

\begin{abstract}
We compare a number of different definitions of structure algebras and TKK constructions for Jordan (super)algebras appearing in the literature. We demonstrate that, for unital superalgebras, all the definitions of the structure algebra and the TKK constructions fall apart into two cases. Moreover, one can be obtained as the Lie superalgebra of superderivations of the other. We also show that, for non-unital superalgebras, more definitions become non-equivalent. As an application, we obtain the corresponding Lie superalgebras for all simple finite dimensional Jordan superalgebras over an algebraically closed field of characteristic zero.
\end{abstract}

\section{INTRODUCTION}

There is an acclaimed principle that associates a 3-graded Lie algebra to a Jordan algebra, as developed by Tits, Kantor and Koecher in three variations, see [Ti, Kan, Ko]. These three constructions have natural analogues for Jordan superalgebras and some also extend to Jordan (super)pairs. The principle behind these three constructions, and further variations appearing in the literature, is loosely referred to as "the" TKK construction.

A common feature of TKK constructions is that, under the appropriate conditions, they associate simple Lie superalgebras to simple Jordan superalgebras or superpairs. They were as such used to classify simple Jordan superalgebras and superpairs, see [Ka2, CK, KMZ, Kan2, Kr], but also to study representations of Jordan superalgebras, see [MZ, Sh, KS]. When the constructions of Tits, Kantor and Koecher are applied to a simple finite dimensional Jordan algebra over the field of complex numbers, they all yield the same Lie algebra, as follows a posteori from the classification. However, if one applies the TKK constructions to more general algebras, they can give different outcomes.

The aim of the paper is to create more structure in this plethora of TKK constructions, by (dis)proving equivalences of some of the definitions under certain conditions and describing concrete links between the different constructions. First we consider the zero component of the 3 -graded Lie (super)algebra associated to a Jordan (super)algebra, which is often referred to as the structure algebra. Then we construct the 3-graded Lie superalgebra out of the structure algebra and the Jordan superalgebra. We refer to this algebra as the TKK algebra.

We consider four definitions of the structure algebra and show that, for unital Jordan superalgebras, they lead to two non-equivalent versions of the structure algebra. For non-unital Jordan superalgebras, all four definitions are non-equivalent. For completeness, we also review two further definitions of structure algebras of unital Jordan superalgebras, with no direct link to TKK constructions, and prove that these are both equivalent to one of the above definitions. One of these definitions also applies to non-unital Jordan superalgebras, and we prove that it is non-equivalent to the previous definitions. 
Then we consider the TKK algebras. First we introduce Kantor's construction. Koecher's construction appears in several forms in the literature, depending on the choice of structure algebra. Finally, the construction by Tits depends on the structure algebra and an auxiliary three-dimensional Lie algebra, which we assume to be $\mathfrak{s l}_{2}$ for now. This yields 5 definitions of TKK superalgebras associated to a Jordan superalgebra $V$, corresponding to constructions of Tits, Koecher and Kantor:

\begin{tabular}{|l|l|l|}
\hline $\operatorname{Ti}\left(V, \operatorname{Inn}(V), \mathfrak{s l}_{2}\right)$ & $\operatorname{Ko}(V)$ & $\operatorname{Kan}(V)$ \\
\hline $\operatorname{Ti}\left(V, \operatorname{Der}(V), \mathfrak{s l}_{2}\right)$ & $\widehat{\operatorname{Ko}}(V)$ & \\
\hline
\end{tabular}

If $V$ is a simple finite dimensional Jordan algebra over the field of complex numbers, it is known that all five Lie algebras are isomorphic. We prove that so long as $V$ is unital, the three Lie superalgebras in the top row are isomorphic. Under the same assumption, the two algebras in the bottom row are then also isomorphic and given by the algebra of derivations of the Lie superalgebras in the top row. For arbitrary $V$, even when finite dimensional, we show that all five algebras can be pairwise non-isomorphic and that the link between bottom and top row through derivations generally fails.

We derive these results for the super case, but they are already pertinent for ordinary Jordan algebras. However, the differences in definitions are more exposed for Jordan superalgebras, as they already appear for finite dimensional simple Jordan superalgebras over the field of complex numbers. Contrary to simple Lie algebras, simple Lie superalgebras can admit outer derivations, and contrary to Jordan algebras, there is a simple finite dimensional Jordan superalgebra which is non-unital.

Therefore we apply our results to obtain a table with all versions of the TKK construction for the simple finite dimensional Jordan superalgebras over an algebraically closed field of characteristic zero. For this, we can rely on the classification of simple Jordan superalgebras in [CK, Ka2] and the calculation of derivations in [Ka1, Sc].

We organise the paper as follows. In Section 2 we introduce some concepts and terminology regarding Jordan superalgebras and superpairs. In Section 3 we investigate the different definitions of the structure algebra. In Section 4 we compare the constructions of Tits, Kantor and Koecher. In Section 5 we study further variations of the Koecher construction, based on the choice of structure algebra. In Section 6 we use the above to list all the versions of the TKK algebras for the finite dimensional simple Jordan superalgebras over an algebraically closed field of characteristic zero. Finally, in Appendix A we introduce the notation for the Lie superalgebras of type $A, P, Q$ and $H$ as used in Section 6 .

\section{JORDAN SUPERALGEBRAS AND SUPERPAIRS}

In the following, we will consider a super vector space $V=V_{\overline{0}} \oplus V_{\overline{1}}$ over a field $\mathbb{K}$. For an element $x$ of $V_{i}$ we write $|x|=i$ for $i \in\{\overline{0}, \overline{1}\}=\mathbb{Z}_{2}$. By $\langle A\rangle$ we will denote the $\mathbb{K}$-linear span of a set $A$. As is customary in the theory of Jordan superalgebras and superpairs, we will always assume that the characteristic of $\mathbb{K}$ is different from 2 and 3. At this stage we make no other assumptions on $\mathbb{K}$. We note furthermore that the main results of section 3,4 and 5 still hold if we replace $\mathbb{K}$ by a ring containing $\frac{1}{2}$ and $\frac{1}{3}$.

\subsection{Jordan superalgebras.}


Definition 2.1. A Jordan superalgebra is a super vector space $V$ equipped with a bilinear product which satisfies

- $V_{i} V_{j} \subset V_{i+j}, \quad i, j \in \mathbb{Z}_{2}$

- $x y=(-1)^{|x||y|} y x \quad$ (commutativity)

- $(-1)^{|x||z|}\left[L_{x}, L_{y z}\right]+(-1)^{|y||x|}\left[L_{y}, L_{z x}\right]+(-1)^{|z||y|}\left[L_{z}, L_{x y}\right]=0 \quad$ (Jordan identity),

for all homogeneous $x, y, z \in V$. Here, the operator $L_{x}: V \rightarrow V$ is defined by $L_{x}(y)=x y$. $A$ Jordan superalgebra $V$ is unital if there exists an element $e \in V$ such that $e x=x=x e$ for all $x \in V$.

We stress that we do not restrict to finite dimensional algebras.

A Jordan superalgebra satisfies the following relation, see [Ka2, Section 1.2],

$$
\left[\left[L_{x}, L_{y}\right], L_{z}\right]=L_{x(y z)}-(-1)^{|x||y|} L_{y(x z)} .
$$

Define the following operators on $V$ :

$$
D_{x, y}:=2 L_{x y}+2\left[L_{x}, L_{y}\right] .
$$

The Jordan triple product is given by

$$
\{x, y, z\}:=D_{x, y} z=2\left((x y) z+x(y z)-(-1)^{|x||y|} y(x z)\right) .
$$

This triple product satisfies the symmetry property

$$
\{x, y, z\}=(-1)^{|x||y|+|y||z|+|x||z|}\{z, y, x\},
$$

and the 5-linear Jordan identity

$$
\begin{aligned}
\{x, y,\{u, v, w\}\} & -\{\{x, y, u\}, v, w\} \\
& =(-1)^{(|x|+|y|)(|u|+|v|)}(-\{u,\{v, x, y\}, w\}+\{u, v,\{x, y, w\}\}) .
\end{aligned}
$$

The 5-linear identity can be rewritten as

$$
\begin{aligned}
{\left[D_{x, y}, D_{u, v}\right] } & =D_{\{x, y, u\}, v}-(-1)^{(|x|+|y|)(|u|+|v|)} D_{u,\{v, x, y\}} \\
& =D_{x,\{y, u, v\}}-(-1)^{(|x|+|y|)(|u|+|v|)} D_{\{u, v, x\}, y} .
\end{aligned}
$$

2.2. Jordan superpairs. A Jordan superpair is a pair of super vector spaces $\left(V^{+}, V^{-}\right)$equipped with two even trilinear products, known as the Jordan triple products,

$$
\{\cdot, \cdot, \cdot\}^{\sigma}: V^{\sigma} \times V^{-\sigma} \times V^{\sigma} \rightarrow V^{\sigma}, \quad \text { for } \quad \sigma \in\{+,-\} .
$$

These triple products satisfy symmetry in the outer variables

$$
\{x, y, z\}^{\sigma}=(-1)^{|x||y|+|y||z|+|z||x|}\{z, y, x\}^{\sigma},
$$

and the 5-linear identity

$$
\begin{aligned}
\left\{x, y,\{u, v, w\}^{\sigma}\right\}^{\sigma} & -\left\{\{x, y, u\}^{\sigma}, v, w\right\}^{\sigma} \\
& =(-1)^{(|x|+|y|)(|u|+|v|)}\left(-\left\{u,\{v, x, y\}^{-\sigma}, w\right\}^{\sigma}+\left\{u, v,\{x, y, w\}^{\sigma}\right\}^{\sigma}\right),
\end{aligned}
$$

for homogeneous $x, z, u, w \in V^{\sigma}$ and $y, v \in V^{-\sigma}$.

We will use the following operators

$$
D_{x, y}: V^{\sigma} \rightarrow V^{\sigma} ; \quad z \mapsto\{x, y, z\}^{\sigma},
$$


for $x \in V^{\sigma}$ and $y \in V^{-\sigma}$.

Example 2.2. By the previous subsection, the doubling of a Jordan superalgebra $V$ gives a Jordan superpair $\left(V^{+}, V^{-}\right):=(V, V)$ with products $\left\{x^{\sigma}, y^{-\sigma}, z^{\sigma}\right\}^{\sigma}:=\{x, y, z\}$ for $\sigma \in\{+,-\}$. Here we use the notation $x^{+}$, resp. $x^{-}$, for an element $x \in V$ interpreted as in $V^{+}$, resp. $V^{-}$. When the context clarifies in which space we interpret $x \in V$, we will leave out the indices.

In the following sections we will often omit the $\sigma$ in the notation for the triple product since it can be derived from the elements it acts on.

\section{Derivations and the structure Algebra}

In this section we show that the (inner) structure algebra of a unital Jordan superalgebra is isomorphic to the algebra of (inner) derivations of the corresponding superpair. We provide counterexamples to both claims when the Jordan superalgebra is non-unital.

\subsection{The structure algebra for a Jordan superalgebra.}

Definition 3.1. Let $V$ be a Jordan superalgebra. An element $D$ in $\operatorname{End}(V)$ is called a derivation of $V$ if

$$
D(x y)=D(x) y+(-1)^{|x||D|} x D(y) .
$$

We use the notation $\operatorname{Der}(V)$ for the space of derivations, and $\operatorname{Inn}(V)$ for the subspace of inner derivations, which is spanned by the operators $\left[L_{x}, L_{y}\right]$ for $x, y \in V$.

The condition on $D \in \operatorname{End}(V)$ to be a derivation is equivalent with

$$
\left[D, L_{x}\right]=L_{D(x)} \quad \text { for all } x \in V .
$$

Hence equation (1) implies that $\left[L_{x}, L_{y}\right]$ is a derivation. One verifies easily that $\operatorname{Der}(V)$ is a subalgebra of $\mathfrak{g l}(V)$. The Jacobi identity on $\mathfrak{g l}(V)$ combined with equation (4), for any derivation $D$, implies that $\operatorname{Inn}(V)$ is an ideal in $\operatorname{Der}(V)$.

We will use the following definition for the structure algebra of Jordan superalgebras, since this is the one that will be required for the Kantor functor. There exist other definitions of the structure algebra in the literature which are not immediately connected to TKK constructions. We will review them in Section 3.4 and show that for unital Jordan superalgebras they are all equivalent to our definition.

Definition 3.2. The structure algebra $\mathfrak{s t r}(V)$ is a subalgebra of $\mathfrak{g l}(V)$, defined as

$$
\mathfrak{s t r}(V)=\left\{L_{x} \mid x \in V\right\}+\operatorname{Der}(V) .
$$

Definition 3.3. The inner structure algebra $\mathfrak{i s t r}(V)$ is a subalgebra of $\mathfrak{g l}(V)$, defined as

$$
\begin{aligned}
\mathfrak{i s t r}(V) & =\left\{L_{x} \mid x \in V\right\}+\operatorname{Inn}(V) \\
& =\left\langle L_{x},\left[L_{x}, L_{y}\right] \mid x, y \in V\right\rangle .
\end{aligned}
$$

By the above, $\mathfrak{i s t r}(V)$ is an ideal in $\mathfrak{s t r}(V)$.

Remark 3.4. For a unital Jordan superalgebra the sum in Definitions 3.2 and 3.3 is a direct sum of super vector spaces:

$$
\mathfrak{s t r}(V)=\left\{L_{x} \mid x \in V\right\} \oplus \operatorname{Der}(V) \text { and } \operatorname{istr}(V)=\left\{L_{x} \mid x \in V\right\} \oplus \operatorname{Inn}(V),
$$

since $D(e)=0$ for all $D$ in $\operatorname{Der}(V)$, while $L_{x}(e)=x$. For non-unital Jordan superalgebras the sums are not necessarily direct, as follows from Example 3.5 and Remark 3.18. 
Example 3.5. Consider the commutative three dimensional algebra $V=\left\langle e_{1}, e_{1}, e_{3}\right\rangle$ with product

$$
e_{1}^{2}=e_{1}, \quad e_{1} e_{2}=\frac{1}{2} e_{2}, \quad e_{2}^{2}=e_{3},
$$

and all other products of basis elements zero. This is the Jordan algebra $\mathcal{J}_{19}$ in [KM, Section 3.3.3]. Because

we conclude that $L_{e_{2}}$ is an element of $\operatorname{Inn}(V)$.

$$
\left[L_{e_{1}}, L_{e_{2}}\right]=-\frac{1}{2} L_{e_{2}}
$$

\subsection{Derivations of Jordan superpairs.}

Definition 3.6. Let $\left(V^{+}, V^{-}\right)$be a Jordan superpair. An element $\mathbb{D}=\left(D^{+}, D^{-}\right) \in \operatorname{End}\left(V^{+}\right) \oplus$ $\operatorname{End}\left(V^{-}\right)$is called a derivation of $\left(V^{+}, V^{-}\right)$if

$$
D^{\sigma}(\{x, y, z\})=\left\{D^{\sigma}(x), y, z\right\}+(-1)^{|x| \mid D^{-\sigma}} \mid\left\{x, D^{-\sigma}(y), z\right\}+(-1)^{(|x|+|y|)\left|D^{\sigma}\right|}\left\{x, y, D^{\sigma}(z)\right\} .
$$

We use the notation $\operatorname{Der}\left(V^{+}, V^{-}\right)$for the space of all derivations of $\left(V^{+}, V^{-}\right)$and the notation $\operatorname{Inn}\left(V^{+}, V^{-}\right)$for the subspace of inner derivations, which is spanned by the operators

$$
\mathbb{D}_{x, y}:=\left(D_{x, y},-(-1)^{|x||y|} D_{y, x}\right), \quad \text { for } \quad x \in V^{+}, y \in V^{-} .
$$

Observe that any derivation $\left(D^{+}, D^{-}\right)$can be written as the sum of derivations where $D^{+}$and $D^{-}$have the same parity. The space $\operatorname{Der}\left(V^{+}, V^{-}\right)$hence inherits a grading from the super vector space $\operatorname{End}\left(V^{+}\right) \oplus \operatorname{End}\left(V^{-}\right)$.

By construction, the space $\operatorname{Der}\left(V^{+}, V^{-}\right)$is a subalgebra of $\mathfrak{g l}\left(V^{+}\right) \oplus \mathfrak{g l}\left(V^{-}\right)$. The operator $\mathbb{D}=$ $\left(D^{+}, D^{-}\right) \in \operatorname{End}\left(V^{+}\right) \oplus \operatorname{End}\left(V^{-}\right)$is a derivation if and only if

$$
\left[D^{\sigma}, D_{x, y}\right]=D_{D^{\sigma}(x), y}+(-1)^{|\mathbb{D}||x|} D_{x, D^{-\sigma}(y)} .
$$

One can then easily verify that $\operatorname{Inn}\left(V^{+}, V^{-}\right)$is an ideal in $\operatorname{Der}\left(V^{+}, V^{-}\right)$.

3.3. Connections. The main result of this section is the following connection between the structure algebra of a unital Jordan superalgebra and the derivations of the associated Jordan superpair in Example 2.2.

Proposition 3.7. For a unital Jordan superalgebra $V$ we have

(1) $\mathfrak{s t r}(V) \cong \operatorname{Der}(V, V)$,

(2) $\operatorname{istr}(V) \cong \operatorname{Inn}(V, V)$.

Remark 3.8. For a unital Jordan algebra, we have that $\mathfrak{s t r}(V)$ is the Lie algebra of the structure group (Section 3.4) and that $\operatorname{Der}(V, V)$ is the Lie algebra of the automorphism group of the Jordan pair $(V, V)$, ([Lo, I.1.4]. Since the structure group is isomorphic to the automorphism group of the Jordan pair, [Lo, Proposition 1.8], we can immediately conclude that $\mathfrak{s t r}(V) \cong$ $\operatorname{Der}(V, V)$.

Remark 3.9. Both parts of the proposition do not extend, as stated, to non-unital Jordan superalgebras. As a counterexample consider again Example 3.5. One can easily check that

$$
\mathfrak{i s t r}(V)=\left\langle D_{x, y} \mid x, y \in V\right\rangle=\left\langle L_{e_{1}}, L_{e_{2}}\right\rangle \text { and } \operatorname{Inn}(V, V)=\left\langle\left(L_{e_{1}},-L_{e_{1}}\right),\left(L_{e_{2}}, 0\right),\left(0, L_{e_{2}}\right)\right\rangle .
$$

Define $A \in \operatorname{End}(V)$ by $A\left(e_{1}\right)=0, A\left(e_{2}\right)=2 e_{2}$ and $A\left(e_{3}\right)=e_{3}$. Then we also obtain

$$
\mathfrak{s t r}(V)=\mathfrak{i s t r}(V)+\langle A\rangle \text { and } \operatorname{Der}(V, V)=\operatorname{Inn}(V, V)+\langle(A, A),(A,-A)\rangle .
$$


Subsection 6.2 also contains a counterexample where $\mathfrak{i s t r}(V) \cong \operatorname{Inn}(V, V)$ but $\mathfrak{s t r}(V) \nsucceq \operatorname{Der}(V, V)$.

Even without the existence of a multiplicative identity $e$, we still have a chain of inclusions if $L_{x}$ is not a derivation for any $x$ in $V$.

Proposition 3.10. For a Jordan superalgebra $V$ for which $L_{x} \notin \operatorname{Der}(V)$, for all $x$ in $V$, we have

$$
\operatorname{Inn}(V, V) \subset \mathfrak{i s t r}(V) \subset \mathfrak{s t r}(V) \subset \operatorname{Der}(V, V) .
$$

Remark 3.11. Examples where the second inclusion is strict can be found in Subsection 6.1 while an example for the third inclusion to be strict can be found in Subsection 6.2.

Now we start the proofs of the propositions.

Lemma 3.12. Let $V$ be a Jordan superalgebra. For $x$ in $V$ and $D$ in $\operatorname{Der}(V)$, we have that

$$
\left(L_{x},-L_{x}\right) \quad \text { and } \quad(D, D)
$$

are elements of $\operatorname{Der}(V, V)$.

Proof. Using the Jordan identity and equation (1), we get for $x, y, z$ in $V$

$$
\begin{aligned}
{\left[L_{x}, D_{y, z}\right] } & =2\left[L_{x}, L_{y z}\right]+2\left[L_{x},\left[L_{y}, L_{z}\right]\right] \\
& =-2(-1)^{|x|(|y|+|z|)}\left[L_{y}, L_{z x}\right]-2(-1)^{|z|(|x|+|y|)}\left[L_{z}, L_{x y}\right]+2 L_{(x y) z}-2(-1)^{|x||y|} L_{y(x z)} \\
& =D_{L_{x}(y), z}-(-1)^{|x||y|} D_{y, L_{x}(z)} .
\end{aligned}
$$

Thus $\left(L_{x},-L_{x}\right)$ satisfies equation (5) and hence belongs to $\operatorname{Der}(V, V)$.

Let $D \in \operatorname{Der}(V)$. By equation (4), the Jacobi identity and the definition of $\operatorname{Der}(V)$, we find

$$
\begin{aligned}
{\left[D, D_{x, y}\right] } & =2\left[D, L_{x y}\right]+2\left[D,\left[L_{x}, L_{y}\right]\right] \\
& =2 L_{D(x y)}-2(-1)^{|D|(|x|+|y|)}\left[L_{x},\left[L_{y}, D\right]\right]-2(-1)^{|y|(|D|+|x|)}\left[L_{y},\left[D, L_{x}\right]\right] \\
& =2 L_{D(x) y}+2\left[L_{D(x)}, L_{y}\right]+2(-1)^{|D||x|} L_{x D(y)}+2(-1)^{|x||D|}\left[L_{x}, L_{D(y)}\right] \\
& =D_{D(x), y}+(-1)^{|x||D|} D_{x, D(y)} .
\end{aligned}
$$

Therefore, also $(D, D)$ satisfies equation (5) and is thus an element of $\operatorname{Der}(V, V)$.

Proof of Proposition 3.10. Since $D_{x, y}=2 L_{x y}+2\left[L_{x}, L_{y}\right]$, the map

$$
\psi: \operatorname{Inn}(V, V) \rightarrow \mathfrak{i s t r}(V) ; \quad \mathbb{D}_{x, y}=\left(D_{x, y},-(-1)^{|x||y|} D_{y, x}\right) \mapsto D_{x, y}
$$

is well-defined and clearly a Lie superalgebra morphism. Assume $D_{x, y}=0$. Then $L_{x y}=-\left[L_{x}, L_{y}\right]$ is a derivation. So by our assumption $L_{x y}=0$, an thus also $D_{y, x}=0$. Therefore $\psi$ is injective.

From the definitions it follows immediately that $\mathfrak{i s t r}(V) \subset \mathfrak{s t r}(V)$. By assumption, $\mathfrak{s t r}(V)$ is a direct sum of $\left\{L_{x} \mid x \in V\right\}$ and $\operatorname{Der}(V)$. Together with Lemma 3.12 this implies that the map

$$
\phi: \mathfrak{s t r}(V) \rightarrow \operatorname{Der}(V, V) ; \quad L_{x}+D \mapsto\left(L_{x}+D,-L_{x}+D\right),
$$

is well-defined. This map is clearly injective. The fact that this is also a Lie superalgebra morphism follows from a direct computation, which finishes the proof. 
The rest of this section is devoted to the proof of Proposition 3.7, so we consider a unital Jordan superalgebra $V$. From Remark 3.4 it follows then that the assumption of Proposition 3.10 is satisfied, so we can use that result. We will also use the following immediate consequences of equation (2),

$$
\frac{1}{2}\{x, e, y\}=x y=L_{x}(y) \quad \text { and } \quad \frac{1}{2}\{e, x, e\}=x .
$$

Consider the map $\sigma$

$$
\sigma: \operatorname{Der}(V, V) \rightarrow \operatorname{Der}(V, V) ; \quad\left(D^{+}, D^{-}\right) \mapsto\left(D^{-}, D^{+}\right) .
$$

Then $\sigma^{2}=\mathrm{id}$ and $\operatorname{Der}(V, V)$ decomposes in two subspaces

$$
\mathfrak{h}:=\{\mathbb{D} \in \operatorname{Der}(V, V) \mid \sigma(\mathbb{D})=\mathbb{D}\} \text { and } \mathfrak{q}:=\{\mathbb{D} \in \operatorname{Der}(V, V) \mid \sigma(\mathbb{D})=-\mathbb{D}\} .
$$

Lemma 3.13. We have

$$
\mathfrak{h}=\left\{\mathbb{D} \in \operatorname{Der}(V, V) \mid D^{-}(e)=0\right\}
$$

Proof. Assume first that $D^{-}(e)=0$. By equation (8), we have

$$
D^{-}(e)=\frac{1}{2} D^{-}\{e, e, e\}=\frac{1}{2}\left\{e, D^{+}(e), e\right\}=D^{+}(e),
$$

and hence also $D^{+}(e)=0$. Then we also get for all $x$ in $V$

$$
2 D^{+}(x)=D^{+}\{e, x, e\}=\left\{e, D^{-}(x), e\right\}=2 D^{-}(x) .
$$

Hence $D^{+}=D^{-}$.

Now assume that $D^{+}=D^{-}$. Equation (8) then implies

$$
D^{-}(e)=\frac{1}{2} D^{-}\{e, e, e\}=\frac{1}{2}\left\{D^{-}(e), e, e\right\}+\frac{1}{2}\left\{e, D^{+}(e), e\right\}+\frac{1}{2}\left\{e, e, D^{-}(e)\right\}=3 D^{-}(e) .
$$

Hence $D^{-}(e)=0$. This concludes the proof.

Lemma 3.14. We have a Lie superalgebra isomorphism

$$
\operatorname{Der}(V) \cong \mathfrak{h}, \quad \text { given by } \quad \phi: \operatorname{Der}(V) \rightarrow \mathfrak{h} ; D \mapsto(D, D) .
$$

Proof. The map $\phi$ is a restriction to $\operatorname{Der}(V)$ of the morphism $\phi: \mathfrak{s t r}(V) \rightarrow \operatorname{Der}(V, V)$ defined in (7). From there we know that it is injective. The image of $\phi$ is clearly contained in $\mathfrak{h}$. To show that $\phi$ is surjective, we let $\mathbb{D}=\left(D^{+}, D^{-}\right)$be an element of $\operatorname{Der}\left(V^{+}, V^{-}\right)$with $D^{+}=D^{-}$, i.e. $\mathbb{D} \in \mathfrak{h}$. Then $D^{-}(e)=0$ by Lemma 3.13. Hence, using equation (8),

$$
\begin{aligned}
D^{+}(x y) & =\frac{1}{2} D^{+}\{x, e, y\} \\
& =\frac{1}{2}\left\{D^{+} x, e, y\right\}+(-1)^{|D \| x|} \frac{1}{2}\left\{x, D^{-} e, y\right\}+(-1)^{|D||x|} \frac{1}{2}\left\{x, e, D^{+} y\right\} \\
& =D^{+}(x) y+(-1)^{|D||x|} x D^{+}(y) .
\end{aligned}
$$

We conclude that $D^{+}=D^{-}$is an element of $\operatorname{Der}(V)$, so $\left(D^{+}, D^{-}\right)$is in the image of $\phi$.

Lemma 3.15. We have an isomorphism of super vector spaces

$$
\left\{L_{x} \mid x \in V\right\} \stackrel{\sim}{\rightarrow} \mathfrak{q}, \quad \text { given by } \quad L_{x} \mapsto\left(L_{x},-L_{x}\right) .
$$


Proof. The assignment $L_{a} \rightarrow\left(L_{a},-L_{a}\right)$ is a restriction to $\left\{L_{x} \mid x \in V\right\}$ of the injective morphism $\phi: \mathfrak{s t r}(V) \rightarrow \operatorname{Der}(V, V)$ considered in (7). Its image is clearly contained in $\mathfrak{q}$. So the map is well-defined and injective. For an element $\mathbb{D}=(D,-D)$ in $\mathfrak{q}$ we claim that $\left(L_{D(e)},-L_{D(e)}\right)=\mathbb{D}$. Indeed, using equation (8), we have

$$
\begin{aligned}
D(x) & =\frac{1}{2} D(\{e, x, e\})=\frac{1}{2}\{D(e), x, e\}-\frac{1}{2}\{e, D(x), e\}+(-1)^{|D \| x|} \frac{1}{2}\{e, x, D(e)\} \\
& =2 D(e) x-D(x),
\end{aligned}
$$

which implies that $D(x)=L_{D(e)}(x)$. This proves surjectivity.

Proof of Proposition 3.7. Consider the injective morphism

$$
\phi: \operatorname{Der}(V) \oplus\left\{L_{x} \mid x \in V\right\} \rightarrow \operatorname{Der}(V, V) ; \quad L_{x}+D \mapsto\left(L_{x}+D,-L_{x}+D\right)
$$

of (7). From Lemmata 3.14 and 3.15 it follows that $\phi$ is also surjective. This proves part (1) of the proposition. For part (2), consider the injective morphism

$$
\psi: \operatorname{Inn}(V, V) \rightarrow \operatorname{istr}(V) ; \quad\left(D_{x, y},-(-1)^{|x||y|} D_{y, x}\right) \mapsto D_{x, y} .
$$

defined in (6). Since

$$
L_{x}=\frac{1}{2} D_{x, e} \text { and }\left[L_{x}, L_{y}\right]=\frac{1}{4}\left(D_{x, y},-(-1)^{|x||y|} D_{y, x}\right),
$$

the map $\psi$ is surjective, which concludes the proof.

3.4. Alternative definitions for the (inner) structure algebra. We review some further definitions appearing in the literature. Set

$$
U_{x, y}: V \rightarrow V ; z \mapsto(-1)^{|y||z|}\{x, z, y\} .
$$

Then for a unital Jordan algebra we define, see [GN, Section 3.1], $\widetilde{\mathfrak{s t r}}(V):=\left\{X \in \mathfrak{g l}(V) \mid U_{X(a), b}+(-1)^{|X||b|} U_{a, X(b)}=X U_{a, b}+(-1)^{|X|(|a|+|b|)} U_{a, b} X^{*}\right.$ for all $\left.a, b \in V\right\}$, where $X^{*}=-X+2 L_{X(e)}$. In the non-super case, this algebra is the Lie algebra of the structure group, see [Ja, Section 9].

In the literature we did not find an explicit definition of the structure algebra for the non-unital case using this approach. However, we will define a natural generalization which for a unital Jordan superalgebra will reduce to $\widetilde{\mathfrak{s t r}}(V)$. So, for $V$ a Jordan superalgebra, define $\mathfrak{s t r}_{w}(V)$ as the Lie superalgebra consisting of the elements $(X, Y) \in \mathfrak{g l}(V) \oplus \mathfrak{g l}(V)^{o p}$ for which

$$
\begin{aligned}
& U_{X(a), b}+(-1)^{|X||a|} U_{a, X(b)}=X U_{a, b}+(-1)^{|Y|(|a|+|b|)} U_{a, b} Y \text { and } \\
& U_{Y(a), b}+(-1)^{|Y||a|} U_{a, Y(b)}=Y U_{a, b}+(-1)^{|X|(|a|+|b|)} U_{a, b} X
\end{aligned}
$$

holds for all $a, b$ in $V$. If $V$ is a Jordan algebra, one can check that $\mathfrak{s t r}_{w}(V)$ is the Lie algebra of the group consisting of pairs of 'weakly structural transformations', as defined in [McC, II.18.2].

Using the equality $U_{x, y}(z)=(-1)^{|y||z|} D_{x, z}(y)$, one finds that the defining conditions of $\mathfrak{s t r}_{w}(V)$ are equivalent with $(X,-Y) \in \operatorname{Der}(V, V)$. So we conclude that $\mathfrak{s t r}_{w}(V) \cong \operatorname{Der}(V, V)$ in full generality.

Lemma 3.16. For a unital Jordan superalgebra $V$, we have

$$
\mathfrak{s t r}_{w}(V) \cong \widetilde{\mathfrak{s t r}}(V) \cong \mathfrak{s t r}(V) .
$$


Proof. Let $X$ be an element of $\widetilde{\mathfrak{s t r}}(V)$. Note that by definition $X$ satisfies equation (9) for $Y=-X+2 L_{X(e)}$. From Lemma 3.12 we know that $\left(L_{X(e)},-L_{X(e)}\right) \in \operatorname{Der}(V, V)$. Combining this, one shows easily that

$$
U_{Y(a), b}+(-1)^{|Y||b|} U_{a, X(b)}=Y U_{a, b}+(-1)^{|X|(|a|+|b|)} U_{a, b} X \text { for all } a, b \in V
$$

holds for $Y=-X+2 L_{X(e)}$. Thus the map $\varphi: \widetilde{\mathfrak{s t r}}(V) \rightarrow \mathfrak{s t r}_{w}(V) ; X \mapsto\left(X,-X+2 L_{X(e)}\right)$ is well-defined. Let $(X, Y) \in \mathfrak{s t r}_{w}(V)$. Then setting $a$ and $b$ equal to the unit $e$ in equation (9) gives us $Y=-X+2 L_{X(e)}$, hence $\varphi$ is an isomorphism. Since $\mathfrak{s t r}_{w}(V) \cong \operatorname{Der}(V, V)$, Proposition 3.7, immediately implies that $\mathfrak{s t r}_{w}(V)$ is also isomorphic to $\mathfrak{s t r}(V)$.

The inner structure algebra is also often defined as the Lie superalgebra spanned by the operators $D_{x, y} \in \operatorname{End}(V)$, see for example [Ja, Section 9], [Sp, Chapter 4] and [GN, Section 3.1]. For this algebra we will use the notation

$$
\widetilde{\mathfrak{i s t r}}(V):=\left\langle D_{x, y} \mid x, y \in V\right\rangle .
$$

Lemma 3.17. For a Jordan superalgebra $V$ for which $L_{x} \notin \operatorname{Der}(V)$, for all $x$ in $V$, we have

$$
\widetilde{\mathfrak{i s t r}}(V) \cong \operatorname{Inn}(V, V) \text {. }
$$

In particular, for a unital Jordan superalgebra, we have

$$
\widetilde{\mathfrak{i s t r}}(V) \cong \mathfrak{i s t r}(V) .
$$

Proof. By assumption $L_{x y} \notin \operatorname{Der}(V)$, so we have that $D_{x, y}=0$ implies $D_{y, x}=0$. Hence the map $\operatorname{Inn}(V, V) \rightarrow \widetilde{\mathfrak{i s t r}(V)} ; \quad\left(D_{x, y},-(-1)^{|x||y|} D_{y, x}\right) \mapsto D_{x, y}$ is bijective. It is also clearly an algebra morphism. This proves the first part of the lemma. Since unital Jordan superalgebras satisfy $L_{x} \notin \operatorname{Der}(v)$, for all $x$ in $V$, Proposition 3.7 immediately implies the second part of the lemma.

Remark 3.18. In the non-unital case we can both have $\widetilde{\mathfrak{i s t r}}(V) \neq \mathfrak{i s t r}(V)$ and $\widetilde{\mathfrak{i s t r}}(V) \neq$ $\operatorname{Inn}(V, V)$. The example in Remark 3.9 is a counterexample for the second part, while a counterexample for the first part is as follows. Consider $V:=t \mathbb{K}[t] /\left(t^{k}\right)$, the algebra of polynomials in the variable $t$ without constant term, modulo the ideal $\left(t^{k}\right)=t^{k} \mathbb{K}[t]$ of polynomials without term in degree lower than $k$ for some $k \in \mathbb{Z}_{>2}$. This is an (associative) Jordan algebra, for the standard multiplication of polynomials, which does not have multiplicative identity. In this example we have $D_{f, g}=2 L_{f g}$, for all $f, g \in V$. We hence find that

$$
\operatorname{Inn}(V, V) \cong \widetilde{\mathfrak{i s t r}}(V)=\operatorname{Span}_{\mathbb{K}}\left\{L_{t^{2}}, L_{t^{3}}, \ldots, L_{t^{k-2}}\right\} .
$$

On the other hand, by definition, we have

$$
\mathfrak{i s t r}(V)=\operatorname{Span}_{\mathbb{K}}\left\{L_{t}, L_{t^{2}}, \ldots, L_{t^{k-2}}\right\} .
$$

As the dimensions of both abelian Lie algebras do not agree, we find $\mathfrak{i s t r}(V) \neq \widetilde{\mathfrak{i s t r}}(V)$. Observe further that $L_{t^{k-2}}$ is an element of $\operatorname{Der}(V)$. Therefore the structure algebra $\mathfrak{s t r}(V)$ also does not have a direct sum decomposition as in Remark 3.4

\section{The Tits-Kantor-Koecher Construction}

In this section, we will study the three different TKK constructions, dating back to Tits, Kantor and Koecher, and show that, for unital Jordan superalgebras, they are equivalent. Again this claim does not extend to non-unital cases. 
4.1. TKK for Jordan superalgebras (Kantor's approach). In [Ka2], Kac uses the "Kantor functor" Kan to classify simple finite dimensional Jordan superalgebras over an algebraically closed field of characteristic zero. This functor is a generalisation to the supercase of the one considered by Kantor in [Kan]. In particular this functor provides a TKK construction, which we review for arbitrary Jordan superalgebras over arbitrary fields.

We associate to a Jordan superalgebra $V$, the 3-graded Lie superalgebra

$$
\begin{aligned}
\operatorname{Kan}(V) & :=\mathfrak{g}=\mathfrak{g}_{-} \oplus \mathfrak{g}_{0} \oplus \mathfrak{g}_{+}, \quad \text { with } \\
\mathfrak{g}_{-}:=V \quad \text { and } \quad \mathfrak{g}_{0} & :=\mathfrak{i s t r}(V)=\left\langle L_{a},\left[L_{a}, L_{b}\right]\right\rangle \subset \operatorname{End}\left(\mathfrak{g}_{-}\right) .
\end{aligned}
$$

Finally, $\mathfrak{g}_{+}$is defined as the subspace $\left\langle P,\left[L_{a}, P\right]\right\rangle$ of $\operatorname{End}\left(\mathfrak{g}_{-} \otimes \mathfrak{g}_{-}, \mathfrak{g}_{-}\right)$, with

$$
P(x, y)=x y \quad \text { and } \quad\left[L_{a}, P\right](x, y):=a(x y)-(a x) y-(-1)^{|x||y|}(a y) x .
$$

Note that $P=-\left[L_{e}, P\right]$ for a unital Jordan superalgebra.

As the notation suggests, $\left[L_{a}, P\right]$ corresponds to the superbracket of $L_{a} \in \mathfrak{g}_{0}$ and $P \in \mathfrak{g}_{+}$. The Lie superbracket is then completely defined by

- $\left[\mathfrak{g}_{-}, \mathfrak{g}_{-}\right]=0=\left[\mathfrak{g}_{+}, \mathfrak{g}_{+}\right]$.

- $[a, x]=a(x)$, for $a \in \mathfrak{g}_{0}, x \in \mathfrak{g}_{-}$.

- $[A, x](y)=A(x, y)$, for $A \in \mathfrak{g}_{+}, x, y \in \mathfrak{g}_{-}$.

- $\left.[a, B](x, y)=a(B(x, y))-(-1)^{|a||B|} B(a(x), y)\right)-(-1)^{|a||B|+|x||y|} B(a(y), x)$, for $a \in \mathfrak{g}_{0}$, $B \in \mathfrak{g}_{+}$and $x, y \in \mathfrak{g}_{-}$.

To verify that $\operatorname{Kan}(V)$ is a Lie superalgebra, one can use the following relations (see Proposition 5.1 in $[\mathrm{CK}]$ )

- $[P, x]=L_{x}$

- $\left[\left[L_{a}, P\right], x\right]=\left[L_{a}, L_{x}\right]-L_{a x}$

- $\left[L_{a},\left[L_{b}, P\right]\right]=-\left[L_{a b}, P\right]$

- $\left[\left[L_{a}, L_{b}\right], P\right]=0$

- $\left[\left[L_{a}, L_{b}\right],\left[L_{c}, P\right]\right]=(-1)^{|b||c|}\left[L_{a(c b)-(a c) b}, P\right]$.

4.2. TKK for Jordan superpairs (Koecher's approach). In [Ko], Koecher defined a product on a triple consisting of two vector spaces and a Lie algebra acting on these vector spaces. This product makes the triple into a 3-graded anti-commutative algebra, which is a Lie algebra if and only if the vector spaces form a Jordan pair and the Lie algebra acts by derivations on the vector spaces. Hence the Koecher construction gives rise to a TKK construction, not only for Jordan algebras, but for Jordan pairs, which is the most natural formulation. Note that, as the concept of Jordan pairs was not yet studied at the time, Koecher did not use this terminology. This TKK construction can be generalised to the supercase, which was for example used by Krutelevich to classify simple finite dimensional Jordan superpairs over an algebraically closed field in characteristic zero in $[\mathrm{Kr}]$.

We associate a 3 -graded Lie superalgebra $\mathrm{Ko}\left(V^{+}, V^{-}\right)$to the Jordan superpair $\left(V^{+}, V^{-}\right)$in the following way. As vector spaces we have

$$
\operatorname{Ko}\left(V^{+}, V^{-}\right)=V^{+} \oplus \operatorname{Inn}\left(V^{+}, V^{-}\right) \oplus V^{-} .
$$


The Lie super bracket on $\operatorname{Ko}\left(V^{+}, V^{-}\right)$is defined by

$$
\begin{aligned}
{[x, u] } & =\mathbb{D}_{x, u} \\
{\left[\mathbb{D}_{x, u}, y\right] } & =\mathbb{D}_{x, u}(y)=\{x, u, y\} \\
{\left[\mathbb{D}_{x, u}, v\right] } & =\mathbb{D}_{x, u}(v)=-(-1)^{|x||u|}\{u, x, v\} \\
{\left[\mathbb{D}_{x, u}, \mathbb{D}_{y, v}\right] } & =\mathbb{D}_{\mathbb{D}_{x, u}(y), v}+(-1)^{(|x|+|u|)|y|} \mathbb{D}_{y, \mathbb{D}_{x, u}(v)} \\
{[x, y] } & =[u, v]=0,
\end{aligned}
$$

for $x, y \in V^{+}, u, v \in V^{-}$. Recall that $\mathbb{D}_{x, u}=\left(D_{x, u},-(-1)^{|x||u|} D_{u, x}\right) \in \operatorname{Inn}\left(V^{+}, V^{-}\right)$.

In case $V$ is a Jordan superalgebra, we simply write $\operatorname{Ko}(V)$ for $\operatorname{Ko}(V, V)$.

Conversely, with each 3-graded Lie superalgebra $\mathfrak{g}=\mathfrak{g}_{-1} \oplus \mathfrak{g}_{0} \oplus \mathfrak{g}_{+1}$ we can associate a Jordan superpair by $\mathcal{J}(\mathfrak{g})=\left(\mathfrak{g}_{+1}, \mathfrak{g}_{-1}\right)$ with the Jordan triple product given by

$$
\left\{x^{\sigma}, y^{-\sigma}, z^{\sigma}\right\}^{\sigma}:=\left[\left[x^{\sigma}, y^{-\sigma}\right], z^{\sigma}\right] .
$$

Definition 4.1. A 3-graded Lie superalgebra $\mathfrak{g}=\mathfrak{g}_{-} \oplus \mathfrak{g}_{0} \oplus \mathfrak{g}_{+}$is called Jordan graded if

$$
\left[\mathfrak{g}_{+}, \mathfrak{g}_{-}\right]=\mathfrak{g}_{0} \text { and } \mathfrak{g}_{0} \cap Z(\mathfrak{g})=0 .
$$

We have the following result by Lemmata 4 and 5 in $[\mathrm{Kr}]$.

Proposition 4.2. For every Jordan superpair $\left(V^{+}, V^{-}\right)$, we have

$$
\mathcal{J}\left(\operatorname{Ko}\left(V^{+}, V^{-}\right)\right) \cong\left(V^{+}, V^{-}\right) .
$$

Let $\mathfrak{g}$ be a Jordan graded Lie superalgebra, then $\operatorname{Ko}(\mathcal{J}(\mathfrak{g})) \cong \mathfrak{g}$.

Note that the main results in $[\mathrm{Kr}]$ are only concerned with finite dimensional pairs, over algebraically closed fields with characteristic zero. However, the mentioned lemmata still hold for arbitrary Jordan superpairs over a field with characteristic different from 2 or 3.

4.3. Connection. The main result of this section is the following proposition, which shows that Kantor's and Koecher's constructions for unital Jordan superalgebras coincide.

Proposition 4.3. For a unital Jordan superalgebra $V$, we have $\operatorname{Kan}(V) \cong \operatorname{Ko}(V)$.

Proof. Let $\operatorname{Kan}(V)=\mathfrak{g}_{+} \oplus \mathfrak{g}_{0} \oplus \mathfrak{g}_{-}$. The relations $[P, a]=L_{a}$ and $\left[\left[L_{a}, P\right], b\right]=\left[L_{a}, L_{b}\right]-L_{a b}$ in Subsection 4.1 imply $\left[\mathfrak{g}_{-}, \mathfrak{g}_{+}\right]=\mathfrak{g}_{0}$. For all $x \in \mathfrak{g}_{0}$, it follows from the definition of the bracket that, if $\left[x, \mathfrak{g}_{-}\right]=0$, then $x=0$. So $\mathfrak{g}_{0} \cap Z(\mathfrak{g})=0$ and $\operatorname{Kan}(V)$ is Jordan graded. Hence Proposition 4.2 implies

$$
\operatorname{Ko}(\mathcal{J}(\operatorname{Kan}(V))) \cong \operatorname{Kan}(V)
$$

Set $\left(V^{+}, V^{-}\right):=\mathcal{J}(\operatorname{Kan}(V))$. Then $V^{-}=V$ and $V^{+}=\left\langle P,\left[L_{a}, P\right] \mid a \in V\right\rangle$. One can check that the map $\phi:\left(V^{+}, V^{-}\right) \rightarrow(V, V)$ defined by

- $\phi(x)=x$ for $x \in V^{-}$,

- $\phi(P)=-\frac{e}{2}$, where $e$ is the unit of $V$,

- $\phi\left(\left[L_{a}, P\right]\right)=\frac{a}{2}$. 
is an isomorphism of Jordan pairs. From this it follows that

$$
\operatorname{Ko}(V)=\operatorname{Ko}(V, V) \cong \operatorname{Ko}(\mathcal{J}(\operatorname{Kan}(V))) \cong \operatorname{Kan}(V),
$$

which proves the proposition.

Remark 4.4. The proposition as stated does not extend to Jordan superalgebras without multiplicative identity $e$. If $V$ is finite dimensional but not unital, we will generally have

$$
\operatorname{dim} \operatorname{Kan}(V)_{+} \neq \operatorname{dim} V=\operatorname{dim} \operatorname{Ko}(V)_{+},
$$

and hence $\operatorname{Kan}(V) \nRightarrow \operatorname{Ko}(V)$. This difference in dimension can for instance be caused by the occurrence of elements of $V$ for which the left multiplication operator is trivial, since this lowers the dimension of $\left\langle P,\left[L_{a}, P\right]\right\rangle$, or by $P \notin\left\langle\left[L_{a}, P\right]\right\rangle$, which raises the dimension.

Another source of counterexamples comes from Jordan superalgebras $V$ which satisfy $\operatorname{Inn}(V, V) \neq$ $\mathfrak{i s t r}(V)$, see e.g. Remark 3.9.

4.4. Tits' approach. There is a third version of the TKK construction, which appeared in [Ti] and historically was the first to appear. In this section, we will give the super version of this construction by Tits.

Consider an arbitrary Jordan superalgebra $V$. Let $\mathcal{D}$ be a Lie superalgebra, containing $\operatorname{Inn}(V)$, with a Lie superalgebra morphism

$$
\psi: \mathcal{D} \rightarrow \operatorname{Der}(V) ; \quad d \mapsto \psi_{d},
$$

such that $\psi$ acts as the identity on the subalgebra $\operatorname{Inn}(V)$. Finally, let $Y$ be an arbitrary threedimensional simple Lie algebra $Y$. For example, for $\mathbb{K}=\mathbb{C}$, we only have $Y \cong \mathfrak{s l}_{2}(\mathbb{C})$ and for $\mathbb{K}=\mathbb{R}$ either $Y \cong \mathfrak{s l}_{2}(\mathbb{R}) \cong \mathfrak{s u}(1,1)$ or $Y \cong \mathfrak{s u}(2)$. Let $\left(y, y^{\prime}\right):=\frac{1}{2} \operatorname{tr}\left(\operatorname{ad}(y) \operatorname{ad}\left(y^{\prime}\right)\right)$ be the Killing form on $Y$.

Then we define a Lie superalgebra

$$
\operatorname{Ti}(V, \mathcal{D}, Y):=\mathcal{D} \oplus(Y \otimes V),
$$

where $\mathcal{D}$ is a subalgebra, and the rest of the multiplication is defined by

$$
\begin{aligned}
{[d, y \otimes v] } & =y \otimes \psi_{d}(v), \\
{\left[y \otimes v, y^{\prime} \otimes v^{\prime}\right] } & =\left(y, y^{\prime}\right)\left[L_{v}, L_{v^{\prime}}\right]+\left[y, y^{\prime}\right] \otimes v v^{\prime},
\end{aligned}
$$

for arbitrary $d \in \mathcal{D}, y, y^{\prime} \in Y$ and $v, v^{\prime} \in V$. For $Y=\mathfrak{s l}_{2}(\mathbb{K})$ we can use the 3-grading on $\mathfrak{s l}_{2}(\mathbb{K})$ to define a 3-grading on $\operatorname{Ti}\left(V, \mathcal{D}, \mathfrak{s l}_{2}(\mathbb{K})\right)$ :

$\operatorname{Ti}\left(V, \mathcal{D}, \mathfrak{s l}_{2}(\mathbb{K})\right)_{-}=\mathfrak{s l}_{2}(\mathbb{K})_{-} \otimes V, \quad \operatorname{Ti}(V, \mathcal{D}, Y)_{0}=\mathcal{D} \oplus\left(\mathfrak{s l}_{2}(\mathbb{K})_{0} \otimes V\right), \quad \operatorname{Ti}\left(V, \mathcal{D}, \mathfrak{s l}_{2}(\mathbb{K})\right)_{+}=\mathfrak{s l}_{2}(\mathbb{K})_{+} \otimes V$.

For unital Jordan superalgebras, this contains, as a special case, Koecher's and hence also Kantor's construction, as we prove in the following proposition.

Proposition 4.5. For a unital Jordan superalgebra $V$ we have

$$
\operatorname{Ti}\left(V, \operatorname{Inn}(V), \mathfrak{s l}_{2}(\mathbb{K})\right) \cong \operatorname{Ko}(V) .
$$

Proof. Consider a $\mathbb{K}$-basis $e, f, h$ of $\mathfrak{s l}_{2}(\mathbb{K})$, such that $[e, f]=h,[h, e]=2 e$ and $[h, f]=-2 f$. For a unital Jordan superalgebra $V$, we have $\operatorname{Inn}(V, V) \cong \mathfrak{i s t r}(V)$, by Proposition 3.7(2).

Then an isomorphism between $\operatorname{Ti}\left(V, \operatorname{Inn}(V), \mathfrak{s l}_{2}(\mathbb{K})\right)$ and $\operatorname{Ko}(V)=V^{+} \oplus \mathfrak{i s t r}(V) \oplus V^{-}$is given by

$$
e \otimes a \mapsto a^{+}, \quad f \otimes a \mapsto a^{-}, \quad h \otimes a \mapsto 2 L_{a}, \quad\left[L_{a}, L_{b}\right] \mapsto\left[L_{a}, L_{b}\right] .
$$


It follows from the definitions that this is a Lie superalgebra morphism.

Remark 4.6. From the proof, it is clear that the proposition still holds for non-unital Jordan superalgebras so long as $\mathfrak{i s t r}(V) \cong \operatorname{Inn}(V, V)$.

Now we consider the opposite direction of the above construction. Let $N$ be a Lie superalgebra and $Y$ a simple Lie algebra of dimension 3. We say that $Y$ acts on $N$ if there is an (even) Lie superalgebra homomorphism from $Y$ to $\operatorname{Der}(N)$. For example, we can define an action of $Y$ on $\operatorname{Ti}(V, \mathcal{D}, Y)$ as follows

$$
y \cdot\left(d+y^{\prime} \otimes v\right)=\left[y, y^{\prime}\right] \otimes v .
$$

Under this action, $\operatorname{Ti}(V, \mathcal{D}, Y)$ viewed as an $Y$-module decomposes as a trivial part given by $\mathcal{D}$ and $\operatorname{dim}(V)$ copies of the adjoint representation.

Now consider an arbitrary Lie superalgebra $N$ with $Y$-action which decomposes as above, viz. as a trivial representation $\mathcal{D}$ and some copies of the adjoint representation,

$$
N=\mathcal{D} \oplus(Y \otimes A),
$$

for some vector space $A$. As a direct generalisation of [Ti], we show that there is a Jordan algebra structure on $A$ where $\mathcal{D}$ acts on $A$ by derivations and $\operatorname{Ti}(V, \mathcal{D}, Y)$ is the inverse of this construction.

Proposition 4.7. Let $N$ be a Lie superalgebra and $Y$ a 3-dimensional simple Lie algebra which acts on $N$ such that $N$ decomposes as $N=\mathcal{D} \oplus(Y \otimes A)$ where $\mathcal{D}$ is a trivial representation and $Y$ the adjoint representation. Then $A$ is a Jordan superalgebra and $\mathcal{D}$ is a superalgebra containing the inner derivations on A equipped with a morphism $\psi: \mathcal{D} \rightarrow \operatorname{Der}(A)$, for which the restriction to the inner derivations is the identity. Furthermore

$$
N \cong \operatorname{Ti}(A, \mathcal{D}, Y)
$$

Proof. Proposition 1 in [Ti] and its proof, which extend trivially to the super case, imply that under these conditions, $\mathcal{D}$ is a subalgebra of $N$, and there are bilinear maps $\alpha(\cdot, \cdot): \mathcal{D} \times A \rightarrow A$, $\langle\cdot, \cdot\rangle: A \times A \rightarrow \mathcal{D}$ and $\mu: A \times A \rightarrow A$, such that

$$
[d, y \otimes a]=y \otimes \alpha(d, a) \quad \text { and } \quad\left[y \otimes a, y^{\prime} \otimes a^{\prime}\right]=\left(y, y^{\prime}\right)\left\langle a, a^{\prime}\right\rangle+\left[y, y^{\prime}\right] \mu\left(a, a^{\prime}\right) .
$$

Furthermore $(A, \mu)$ is a Jordan superalgebra and $d \mapsto \alpha(d, \cdot)$ is a Lie superalgebra morphism $\phi: \mathcal{D} \rightarrow \operatorname{Der}(A)$. Finally, by equation (2.6) in [Ti], we have

$$
\phi(\langle a, b\rangle)=\left[L_{a}, L_{b}\right] .
$$

Comparison with the definition of $\operatorname{Ti}(A, \mathcal{D}, Y)$ concludes the proof.

\section{Further TKK CONSTRUCtions}

In this section we consider variations of the TKK constructions for a Jordan superalgebra $V$, which also appear in the literature, by using $\mathfrak{s t r}(V)$ and $\operatorname{Der}(V, V)$, instead of $\mathfrak{i s t r}(V)$ and $\operatorname{Inn}(V, V)$. 
5.1. Definition. The Lie superalgebra $\operatorname{Ti}\left(V, \mathcal{D}, \mathfrak{s l}_{2}\right)$ had more freedom compared to the constructions by Kantor and Koecher, due to the choice of $\mathcal{D}$. Also in the Koecher construction, we can replace $\operatorname{Inn}\left(V^{+}, V^{-}\right)$by any Lie superalgebra containing $\operatorname{Inn}\left(V^{+}, V^{-}\right)$with a morphism to $\operatorname{Der}\left(V^{+}, V^{-}\right)$which restricts to the identity on the inner derivations. For example, we can set $\mathfrak{g}_{0}=\operatorname{Der}\left(V^{+}, V^{-}\right)$in the TKK construction of Section 4.2. This gives a 3-graded Lie superalgebra

$$
\widetilde{\mathrm{Ko}}\left(V^{+}, V^{-}\right)=V^{+} \oplus \operatorname{Der}\left(V^{+}, V^{-}\right) \oplus V^{-},
$$

see [GN] for more details. Remark that, by construction, $\operatorname{Ko}\left(V^{+}, V^{-}\right)$is an ideal in $\widetilde{\operatorname{Ko}}\left(V^{+}, V^{-}\right)$. We will again use the notation $\widetilde{\mathrm{Ko}}(V)$ for $\widetilde{\mathrm{Ko}}(V, V)$. In Subsection 5.3 , we will prove that $\widetilde{\mathrm{Ko}}(V)$ is the superalgebra of derivations of $\operatorname{Ko}(V)$ for unital Jordan superalgebras. We can also relate $\widetilde{\mathrm{Ko}}(V)$ to the Tits' construction in Subsection 4.4 as follows.

Lemma 5.1. For a unital Jordan superalgebra $V$, we have

$$
\widetilde{\mathrm{Ko}}(V) \cong \operatorname{Ti}\left(V, \operatorname{Der}(V), \mathfrak{s l}_{2}(\mathbb{K})\right) .
$$

This will be proved in greater generality in Subsection 5.2.

5.2. Comparison of further TKK constructions. Let $\mathcal{D}$ be a Lie superalgebra containing $\operatorname{Inn}(V)$ with a morphism $\psi$ to $\operatorname{Der}(V)$ such that $\psi_{\mid \operatorname{Inn}(V)}=$ id. Define the Lie superalgebra

$$
\widetilde{\mathcal{D}}:=\mathcal{D} \oplus\left\{L_{x} \mid x \in V\right\},
$$

where $\mathcal{D}$ is a subalgebra of $\widetilde{\mathcal{D}}$, the product of $L_{x}$ and $L_{y}$ is given by $\left[L_{x}, L_{y}\right]$ interpreted via the embedding of $\operatorname{Inn}(V)$ in $\mathcal{D}$, and

$$
\left[D, L_{x}\right]:=L_{\psi(D) x} \quad \text { for } D \in \mathcal{D}, x \in V .
$$

Set

$$
\widetilde{\psi}: \widetilde{\mathcal{D}} \rightarrow \operatorname{Der}(V, V) ; \quad D+L_{x} \mapsto\left(\psi(D)+L_{x}, \psi(D)-L_{x}\right) .
$$

From Lemma 3.12, it follows that this map is well defined, while from the definition of the bracket on $\widetilde{\mathcal{D}}$ it follows that it is a Lie superalgebra morphism. The morphism $\widetilde{\psi}$ yields an action of $\widetilde{\mathcal{D}}$ on $V^{+}$and $V^{-}$, which allows us to define a TKK construction similar to the Koecher construction in Subsection 4.2. Concretely, the bracket on

$$
\mathrm{Ko}_{\mathcal{D}}(V):=V \oplus \widetilde{\mathcal{D}} \oplus V
$$

is given by

$$
\begin{array}{r}
{[x, u]=2 L_{x u}+2\left[L_{x}, L_{u}\right], \quad[d, x]=\widetilde{\psi}(d) x, \quad[d, u]=\widetilde{\psi}(d) u,} \\
{\left[d_{1}, d_{2}\right]=\left[d_{1}, d_{2}\right]_{\widetilde{\mathcal{D}}}, \quad[x, y]=0=[u, v],}
\end{array}
$$

for $x, y$ in $V^{+}, u, v$ in $V^{-}, d, d_{1}, d_{2}$ in $\widetilde{D}$ and $[\cdot, \cdot]_{\widetilde{\mathcal{D}}}$ the product in $\widetilde{\mathcal{D}}$.

Proposition 5.2. Consider a (not necessarily unital) Jordan superalgebra $V$ and a Lie superalgebra $\mathcal{D}$ as above. We have an isomorphism of Lie superalgebras

$$
\operatorname{Ti}\left(V, \mathcal{D}, \mathfrak{s l}_{2}(\mathbb{K})\right) \cong \operatorname{Ko}_{\mathcal{D}}(V) .
$$

Proof. The following generalisation of the map used in Proposition 4.5

$$
e \otimes a \mapsto a^{+}, \quad f \otimes a \mapsto a^{-}, \quad h \otimes a \mapsto 2 L_{a}, \quad D \mapsto D,
$$

is an isomorphism between $\operatorname{Ti}\left(V, \mathcal{D}, \mathfrak{s l}_{2}(\mathbb{K})\right)$ and $\operatorname{Ko}_{\mathcal{D}}(V)$. 
The case $\mathcal{D}=\operatorname{Inn}(V)$ yields

$$
\operatorname{Ti}\left(V, \operatorname{Inn}(V), \mathfrak{s l}_{2}(\mathbb{K})\right) \cong \operatorname{Ko}_{\operatorname{Inn}(V)}(V),
$$

with $\widetilde{\operatorname{Inn}(V)}=\left\{L_{x} \mid x \in V\right\} \oplus \operatorname{Inn}(V)$. This is a generalisation of Proposition 4.5 to the non-unital case.

Note that if there exists an $x \in V$, such that $L_{x}$ is in $\operatorname{Der}(V)$, then $\widetilde{\operatorname{Der}(V)}$ contains two copies of $L_{x}$, one in $\operatorname{Der}(V)$ and one in $\left\{L_{x} \mid x \in V\right\}$. The isomorphism between $\widetilde{\operatorname{Der}(V)}$ and $\operatorname{Der}(V, V)$ maps the first to $\left(L_{x}, L_{x}\right)$, while the second copy gets mapped to $\left(L_{x},-L_{x}\right)$. For unital Jordan superalgebras we have canonical isomorphisms $\widetilde{\operatorname{Inn}(V)} \cong \mathfrak{i s t r}(V)$ and $\widetilde{\operatorname{Der}(V)} \cong \mathfrak{s t r}(V)$, by Remark 3.4, and thus $\operatorname{Ko}_{\operatorname{Inn}(V)}(V)=\operatorname{Ko}(V)$ and $\operatorname{Ko}_{\operatorname{Der}(V)}(V)=\widetilde{\operatorname{Ko}}(V)$. Hence we find that Proposition 5.2 implies Lemma 5.1.

Remark 5.3. Let $\mathfrak{g}$ be an arbitrary 3 -graded Lie superalgebra and set $\left(V^{+}, V^{-}\right)=\mathcal{J}(\mathfrak{g})$. Then we have a morphism of Lie superalgebras

$$
\mathfrak{g}_{0} \rightarrow \operatorname{Der}\left(V^{+}, V^{-}\right) ; x \mapsto\left(\left.\operatorname{ad}_{x}\right|_{\mathfrak{g}_{+}},\left.\operatorname{ad}_{x}\right|_{\mathfrak{g}_{-}}\right),
$$

and its kernel $I$ is an ideal in $\mathfrak{g}_{0}$ and by construction even in $\mathfrak{g}$. By definition of $\widetilde{\operatorname{Ko}}\left(V^{+}, V^{-}\right)$, we have an embedding of $\mathfrak{g} / I$ into $\widetilde{\mathrm{Ko}}\left(V^{+}, V^{-}\right)$. If $\mathfrak{g}=\mathrm{Ti}\left(V, \mathcal{D}, \mathfrak{s l}_{2}\right)$ for a unital Jordan superalgebra $V$, then one can easily check that $I=0$ (and thus $\mathcal{D} \subseteq \operatorname{Der}(V)$ ) is equivalent with the condition that the only ideal of $\mathfrak{g}$ contained in $\mathcal{D}$ is the zero ideal.

Another "universality property" of $\widetilde{\mathrm{Ko}}\left(V^{+}, V^{-}\right)$will be discussed in Subsection 5.4.

\subsection{Outer derivations.}

Definition 5.4 (See $[\mathrm{AMR}]$ ). For a Lie superalgebra $\mathfrak{g}$, denote the Lie superalgebra of derivations by $\operatorname{Der}(\mathfrak{g})$. The inner derivations $\operatorname{Inn}(\mathfrak{g})=\left\{\operatorname{ad}_{X} \mid X \in \mathfrak{g}\right\}$ form an ideal isomorphic to the quotient of $\mathfrak{g}$ by its centre. The Lie superalgebra of outer derivations is $\operatorname{Out}(\mathfrak{g})=\operatorname{Der}(\mathfrak{g}) / \operatorname{Inn}(\mathfrak{g})$.

An extension $\mathfrak{e}$ of a Lie superalgebra $\mathfrak{g}$ over a Lie superalgebra $\mathfrak{h}$ is a Lie superalgebra $\mathfrak{e}$ such that the following is a short exact sequence:

$$
0 \rightarrow \mathfrak{h} \rightarrow \mathfrak{e} \rightarrow \mathfrak{g} \rightarrow 0
$$

In particular $\mathfrak{h}$ is an ideal in $\mathfrak{e}$.

Let $\mathfrak{h}$ be a Lie superalgebra with trivial centre. Then we will freely use the isomorphism between the space of extensions of $\mathfrak{g}$ over $\mathfrak{h}$, and the space of Lie superalgebra morphisms $\mathfrak{g} \rightarrow \operatorname{Out}(\mathfrak{h})$, see e.g. Corollary 8 in $[\mathrm{AMR}]$.

The main result of this section is the following proposition.

Proposition 5.5. For a unital Jordan superalgebra $V$, we have

$$
\widetilde{\operatorname{Ko}}(V) \cong \operatorname{Der}(\operatorname{Ko}(V)) \text {, }
$$

and thus

$$
\widetilde{\mathrm{Ko}}(V) / \mathrm{Ko}(V) \cong \mathfrak{s t r}(V) / \mathfrak{i s t r}(V) \cong \operatorname{Out}(\mathrm{Ko}(V)) .
$$

Remark 5.6. Again the assumption of a multiplicative identity is essential for this proposition. A counterexample of the statement for non-unital Jordan superalgebras is given in Subsection 6.2. 
Remark 5.7. For any $\mathbb{Z}$-graded Lie superalgebra, the Lie superalgebra $\operatorname{Der}(\mathfrak{g}) \subset \operatorname{End}_{\mathbb{K}}(\mathfrak{g})$ is $\mathbb{Z}$ graded by construction. The endomorphisms in $\operatorname{Der}(\mathfrak{g})_{i}$ map elements in $\mathfrak{g}_{j}$ to elements in $\mathfrak{g}_{i+j}$. Clearly $\operatorname{Inn}(\mathfrak{g})$ is then a graded ideal in $\operatorname{Der}(\mathfrak{g})$, so that $\operatorname{Out}(\mathfrak{g})$ is also $\mathbb{Z}$-graded. In particular, when $\mathfrak{g}$ is 3 -graded then $\operatorname{Der}(\mathfrak{g})$ and $\operatorname{Out}(\mathfrak{g})$ will be 5 -graded.

The following reformulation of Proposition 5.5 holds for arbitrary Jordan superpairs and thus $a$ fortiori also for non-unital Jordan superalgebras.

Proposition 5.8. For a Jordan superpair $\left(V^{+}, V^{-}\right)$, we have

$$
\widetilde{\operatorname{Ko}}\left(V^{+}, V^{-}\right) / \operatorname{Ko}\left(V^{+}, V^{-}\right) \cong \operatorname{Der}\left(V^{+}, V^{-}\right) / \operatorname{Inn}\left(V^{+}, V^{-}\right) \cong \operatorname{Out}\left(\operatorname{Ko}\left(V^{+}, V^{-}\right)\right)_{0} .
$$

In particular, for a (non-unital) Jordan superalgebra $V$ we have that $\widetilde{\mathrm{Ko}}(V)$ is the extension over $\operatorname{Ko}(V)$ of $\operatorname{Out}(\operatorname{Ko}(V))_{0}$ corresponding to the embedding $\operatorname{Out}(\operatorname{Ko}(V))_{0} \hookrightarrow \operatorname{Out}(\operatorname{Ko}(V))$.

The rest of the subsection is devoted to the proofs of Propositions 5.5 and 5.8.

Lemma 5.9. We have a Lie superalgebra isomorphism

$$
\phi: \operatorname{Der}\left(V^{+}, V^{-}\right) \stackrel{\sim}{\rightarrow} \operatorname{Der}\left(\operatorname{Ko}\left(V^{+}, V^{-}\right)\right)_{0},\left.\quad x \mapsto \operatorname{ad}_{x}\right|_{\mathrm{Ko}\left(V^{+}, V^{-}\right)} .
$$

Proof. As $\operatorname{Ko}\left(V^{+}, V^{-}\right)$is an ideal in $\widetilde{\operatorname{Ko}}\left(V^{+}, V^{-}\right)$and $\operatorname{Der}\left(V^{+}, V^{-}\right) \subset \widetilde{\mathrm{Ko}}\left(V^{+}, V^{-}\right)$is the zero component of the $\mathbb{Z}$-grading, the map $\phi$ is well-defined. By construction it is an injective Lie superalgebra morphism.

Now let $D$ be a $\mathbb{Z}$-grading preserving derivation of $\operatorname{Ko}\left(V^{+}, V^{-}\right)$, then $\left(\left.D\right|_{V^{+}},\left.D\right|_{V^{-}}\right)$is an element of $\operatorname{Der}\left(V^{+}, V^{-}\right)$since, using the definition of the bracket on $\operatorname{Ko}\left(V^{+}, V^{-}\right)$in Subsection 4.2, we find

$$
\begin{aligned}
D(\{x, y, z\})=D([[x, y], z]) & =[[D(x), y], z]+(-1)^{|x||D|}[[x, D(y)], z]+(-1)^{(|x|+|y|)|D|}[[x, y], D(z)] \\
& =\{D(x), y, z\}+(-1)^{|x||D|}\{x, D(y), z\}+(-1)^{(|x|+|y|)|D|}\{x, y, D(z)\} .
\end{aligned}
$$

One can check that

$$
D=\operatorname{ad}_{\left(\left.D\right|_{V^{+}},\left.D\right|_{V^{-}}\right)} \text {and } x=\left(\left.\operatorname{ad}_{x}\right|_{V^{+}},\left.\operatorname{ad}_{x}\right|_{V^{-}}\right) .
$$

So we have indeed $\operatorname{Der}\left(V^{+}, V^{-}\right) \cong \operatorname{Der}\left(\operatorname{Ko}\left(V^{+}, V^{-}\right)\right)_{0}$ as Lie superalgebras.

Using this lemma, we can immediately prove Proposition 5.8.

Proof of Proposition 5.8. The first isomorphism follows immediately from Koecher's construction in Subsection 4.2. Furthermore, since the intersection of the centre of $\operatorname{Ko}\left(V^{+}, V^{-}\right)$with $\mathrm{Ko}\left(V^{+}, V^{-}\right)_{0}$ is trivial, we have

$$
\operatorname{Inn}\left(\operatorname{Ko}\left(V^{+}, V^{-}\right)\right)_{0} \cong \operatorname{Ko}\left(V^{+}, V^{-}\right)_{0}=\operatorname{Inn}\left(V^{+}, V^{-}\right) .
$$

Hence, from Lemma 5.9 we conclude that $\operatorname{Der}\left(V^{+}, V^{-}\right) / \operatorname{Inn}\left(V^{+}, V^{-}\right) \cong \operatorname{Out}\left(\operatorname{Ko}\left(V^{+}, V^{-}\right)\right)_{0}$.

To prove Proposition 5.5, we will show that, for a unital Jordan superalgebra $V$, all outer derivations of $\operatorname{Ko}(V)$ are grading preserving for the 3-grading we consider. This is not true for non-unital algebras, see Subsection 6.2.

Lemma 5.10. For a unital Jordan superalgebra $V$, we have

$$
\operatorname{Der}(\operatorname{Ko}(V))_{-2}=0=\operatorname{Der}(\operatorname{Ko}(V))_{2} .
$$


Proof. Let $D \in \operatorname{Der}(\operatorname{Ko}(V))_{2}$. First remark that $D$ acts trivially on $\operatorname{Ko}(V)_{0}$ and $\operatorname{Ko}(V)_{1}$. We will show that it must also acts trivially on $\operatorname{Ko}(V)_{-1}$. To use the definition of $\operatorname{Ko}(V)$ we use the Jordan superpair $\left(V^{+}, V^{-}\right):=(V, V)$. For $x \in V$, we use the notation $x^{+}$and $x^{-}$, as in Example 2.2. We find, using the definition of the bracket on $\operatorname{Ko}(V)$ and the property $D\left(\operatorname{Ko}(V)_{0}\right)=0$, that

$$
D\left(e^{-}\right)=\frac{1}{2} D\left(\left[e^{-}, \mathbb{D}_{e, e}\right]\right)=\frac{1}{2}\left[D\left(e^{-}\right), \mathbb{D}_{e, e}\right]+\frac{1}{2}\left[e^{-}, D\left(\mathbb{D}_{e, e}\right)\right]=\frac{1}{2}\left[D\left(e^{-}\right), \mathbb{D}_{e, e}\right]=-D\left(e^{-}\right) .
$$

Hence $D\left(e^{-}\right)=0$, which then implies that

$$
D\left(x^{-}\right)=\frac{1}{2} D\left(\left[e^{-}, \mathbb{D}_{x, e}\right]\right)=\frac{1}{2}\left[D\left(e^{-}\right), \mathbb{D}_{x, e}\right]+\frac{1}{2}\left[e^{-}, D\left(\mathbb{D}_{x, e}\right)\right]=0 .
$$

We conclude that $D=0$ for all $D \in \operatorname{Der}(\operatorname{Ko}(V))_{2}$. The proof that $\operatorname{Der}(\operatorname{Ko}(V))_{-2}=0$ is completely similar.

Lemma 5.11. For a unital Jordan superalgebra $V$, we have isomorphisms

$$
V \stackrel{\sim}{\rightarrow} \operatorname{Der}(\operatorname{Ko}(V))_{1} ; \quad x \mapsto \operatorname{ad}_{x^{+}} \text {and } \quad V \stackrel{\sim}{\rightarrow} \operatorname{Der}(\operatorname{Ko}(V))_{-1} ; \quad x \mapsto \operatorname{ad}_{x^{-}},
$$

as super vector spaces.

Proof. Let $x^{+}$be an element of $\operatorname{Ko}(V)_{1}=V^{+}$, then $\operatorname{ad}_{x^{+}} \in \operatorname{Der}(\operatorname{Ko}(V))_{1}$. With an element $D$ in $\operatorname{Der}(\operatorname{Ko}(V))_{1}$ we can associate the element $-\frac{1}{2} D\left(\mathbb{D}_{e, e}\right) \in V^{+}$. We will now show that $x^{+} \mapsto \operatorname{ad}_{x^{+}}$ and $D \mapsto-\frac{1}{2} D\left(\mathbb{D}_{e, e}\right)$ are each others inverse. This follows from

$$
-\frac{1}{2} \operatorname{ad}_{x^{+}}\left(\mathbb{D}_{e, e}\right)=-\frac{1}{2}\left[x^{+}, \mathbb{D}_{e, e}\right]=x^{+}
$$

and the following three calculations, for arbitrary $x, y \in V$,

$$
\begin{aligned}
-\frac{1}{2} \operatorname{ad}_{D\left(\mathbb{D}_{e, e}\right)}\left(y^{-}\right) & =-\frac{1}{2}\left[D\left(\mathbb{D}_{e, e}\right), y^{-}\right]=-\frac{1}{2} D\left(\left[\mathbb{D}_{e, e}, y^{-}\right]\right)+\frac{1}{2}\left[\mathbb{D}_{e, e}, D\left(y^{-}\right)\right]=D\left(y^{-}\right) \\
-\frac{1}{2} \operatorname{ad}_{D\left(\mathbb{D}_{e, e}\right)}\left(L_{x}\right) & =-\frac{1}{2}\left[D\left(\mathbb{D}_{e, e}\right), L_{x}\right]=-\frac{1}{2} D\left(\left[\mathbb{D}_{e, e}, L_{x}\right]\right)+\frac{1}{2}\left[\mathbb{D}_{e, e}, D\left(L_{x}\right)\right]=D\left(L_{x}\right) \\
-\frac{1}{2} \operatorname{ad}_{D\left(\mathbb{D}_{e, e}\right)}\left(\left[L_{x}, L_{y}\right]\right) & =-\frac{1}{2}\left[D\left(\mathbb{D}_{e, e}\right),\left[L_{x}, L_{y}\right]\right]=-\frac{1}{2} D\left(\left[\mathbb{D}_{e, e},\left[L_{x}, L_{y}\right]\right]\right)+\frac{1}{2}\left[\mathbb{D}_{e, e}, D\left(\left[L_{x}, L_{y}\right]\right)\right] \\
& =D\left(\left[L_{x}, L_{y}\right]\right) .
\end{aligned}
$$

We conclude that $V \cong \operatorname{Der}(\operatorname{Ko}(V))_{1}$. Similarly $\operatorname{Ko}(V)_{-1} \rightarrow \operatorname{Der}(\operatorname{Ko}(V))_{-1} ; x^{-} \mapsto \operatorname{ad}_{x^{-}}$is an isomorphism with inverse $D \mapsto \frac{1}{2} D\left(\mathbb{D}_{e, e}\right)$.

Proof of Proposition 5.5. Consider the following morphism of Lie superalgebras

$$
\widetilde{\mathrm{Ko}}(V) \rightarrow \operatorname{Der}(\mathrm{Ko}(V)) ;\left.x \mapsto \operatorname{ad}_{x}\right|_{\mathrm{Ko}(V)} .
$$

Combining Lemmata 5.9, 5.10 and 5.11, we see that this is an isomorphism.

5.4. Alternative construction. The construction of $\widetilde{\mathrm{Ko}}\left(V^{+}, V^{-}\right)$starting from $\operatorname{Ko}\left(V^{+}, V^{-}\right)$in Proposition 5.8 fits into a more general construction. In [BDS, Section 4.1], the authors start from an arbitrary $(2 n+1)$-graded Lie superalgebra $\mathcal{L}=\bigoplus_{i \in \mathbb{Z}} \mathcal{L}_{i}$ (strictly speaking only Lie algebras are considered, but the procedure carries over naturally to the super case). Then [BDS, Construction 4.1.2] constructs an extension $\overline{\mathcal{L}}$ over $\mathcal{L}$, which is again a $(2 n+1)$-graded Lie superalgebra which satisfies $\overline{\mathcal{L}}_{i}=\mathcal{L}_{i}$ if $i \neq 0$. 
It is not difficult to show that in the case of a 3 -graded Lie superalgebra $\mathcal{L}$ we have $\overline{\mathcal{L}_{0}}=$ $\operatorname{Der}\left(\mathcal{L}^{+}, \mathcal{L}^{-}\right)$and hence

$$
\overline{\mathcal{L}}=\widetilde{\operatorname{Ko}}\left(\mathcal{L}^{+}, \mathcal{L}^{-}\right) \quad \text { with } \quad\left(\mathcal{L}^{+}, \mathcal{L}^{-}\right):=\mathcal{J}(\mathcal{L})
$$

the Jordan superpair associated with $\mathcal{L}$ in Subsection 4.2. In other words,

$$
\widetilde{\mathrm{Ko}}\left(V^{+}, V^{-}\right) \cong \overline{\mathrm{Ko}\left(V^{+}, V^{-}\right)}
$$

This reveals a universality principle behind $\widetilde{\mathrm{Ko}}\left(V^{+}, V^{-}\right)$, as the construction of $\overline{\mathcal{L}}$ starting from $\mathcal{L}$ in [BDS] does not depend on $\mathcal{L}_{0}$.

An interesting consequence of [BDS, Lemma 4.1.3] is then

$$
\operatorname{Out}\left(\widetilde{\mathrm{Ko}}\left(V^{+}, V^{-}\right)\right)=0,
$$

for arbitrary Jordan superpairs $\left(V^{+}, V^{-}\right)$, so also for arbitrary (unital or non-unital) Jordan superalgebras.

\section{EXAMPLES}

In this section, we use the results of the previous sections to calculate $\widetilde{\mathrm{Ko}}(V)$ for $V$ any finite dimensional simple Jordan superalgebra over an algebraically closed field of characteristic zero. We assume these conditions on the ground field for the entire section.

6.1. Unital finite dimensional simple Jordan superalgebras. A complete list of unital finite dimensional simple Jordan superalgebras $V$ and the corresponding $\operatorname{Kan}(V)$ is given in [Ka2, CK]. This gives us $\operatorname{Ko}(V)$ and $\operatorname{Ti}\left(V, \operatorname{Inn}(V), \mathfrak{s l}_{2}\right)$, by Propositions 4.3 and 4.5. For the Jordan superalgebras we use the notation of [CK], where also the definitions can be found. We introduce our convention for the notation of Lie superalgebras in Appendix A. In [Ka1, Theorem 5.1.2] and [Sc, Chapter III, Proposition 3], Der $(\mathfrak{g})$ is calculated for any simple finite dimensional Lie superalgebra $\mathfrak{g}$. Together with Proposition 5.5 and Lemma 5.1, this gives us $\widetilde{\operatorname{Ko}}(V) \cong \operatorname{Ti}\left(V, \operatorname{Der}(V), \mathfrak{s l}_{2}\right)$, leading to the following table.

\begin{tabular}{|c|c|c|c|}
\hline$V$ & $\widehat{K o}(V)$ & $\widetilde{K o}(V)$ & Remarks \\
\hline \hline$g l(m, n)_{+}$ & $\mathfrak{s l}(2 m \mid 2 n)$ & & $m \neq n$ \\
\hline$g l(m, m)_{+}$ & $\mathfrak{p} \mathfrak{s l}(2 m \mid 2 m)$ & $\mathfrak{p g r l}(2 m \mid 2 m)$ & $m>1$ \\
\hline$o s p(m, 2 n)_{+}$ & $\mathfrak{o s p}(4 n \mid 2 m)$ & & $(n, m) \neq(1,0)$ \\
\hline$(m-3,2 n)_{+}$ & $\mathfrak{o s p}(m \mid 2 n)$ & & $m \geq 3,(m, 2 n) \neq(4,0)$ \\
\hline$p(n)_{+}$ & $\mathfrak{s p \mathfrak { e }}(2 n)$ & $\mathfrak{p} \mathfrak{p}(2 n)$ & $n>1$ \\
\hline$q(n)_{+}$ & $\mathfrak{p s q}(2 n)$ & $\mathfrak{p q}(2 n)$ & $n>1$ \\
\hline$D_{t}$ & $D(2,1, t)$ & & $t \notin\{0,-1\}$ \\
\hline$E$ & $E(7)$ & & \\
\hline$F$ & $F(4)$ & & $n \geq 5$ \\
\hline$J P(0, n-3)$ & $H(0, n)=H(n)$ & $\mathbb{K} C \ltimes \widetilde{H}(n)$ & \\
\hline$g l(1,1)_{+}$ & $\mathfrak{p} \mathfrak{s l}(2 \mid 2)$ & $D(2,1,-1)$ & \\
\hline
\end{tabular}

When $\widetilde{\operatorname{Ko}}(V)$ is isomorphic to $\operatorname{Ko}(V)$, we only wrote it once.

Taking the zero component of the 3 -graded algebras in the above table gives us $\operatorname{istr}(V) \cong$ $\operatorname{Inn}(V, V)$ and $\mathfrak{s t r}(V) \cong \operatorname{Der}(V, V)$. These are listed in the following table, where the same restrictions on the indices are assumed as in the previous table. 


\begin{tabular}{|c|c|c|}
\hline$V$ & $\mathfrak{i s t r}(V)$ & $\mathfrak{s t r}(V)$ \\
\hline \hline$g l(m, n)_{+}$ & $\mathfrak{s l}(m \mid n) \oplus \mathfrak{s l}(m \mid n) \oplus \mathbb{K}$ & \\
\hline$g l(m, m)_{+}$ & $s(\mathfrak{g l}(m \mid m) \oplus \mathfrak{g l}(m \mid m)) /\left\langle I_{4 m}\right\rangle$ & $(\mathfrak{g l}(m \mid m) \oplus \mathfrak{g l}(m \mid m)) /\left\langle I_{4 m}\right\rangle$ \\
\hline$o s p(m, 2 n)_{+}$ & $\mathfrak{g l}(2 n \mid m)$ & \\
\hline$(m-3,2 n)_{+}$ & $\mathfrak{o s p}(m-2 \mid 2 n) \oplus \mathbb{K}$ & $(\mathfrak{q}(n) \oplus \mathfrak{q}(n)) /\left\langle I_{4 n}\right\rangle$ \\
\hline$p(n)_{+}$ & $\mathfrak{s l}(n \mid n)$ & \\
\hline$q(n)_{+}$ & $s(\mathfrak{q}(n) \oplus \mathfrak{q}(n)) /\left\langle I_{4 n}\right\rangle$ & \\
\hline$D_{t}$ & $\mathfrak{s l}(2 \mid 1) \oplus \mathbb{K} \cong \mathfrak{o} \mathfrak{p} \mathfrak{p}(2 \mid 2) \oplus \mathbb{K}$ & $E(6) \oplus \mathbb{K}$ \\
\hline$E$ & $\mathfrak{o s p}(2 \mid 4) \oplus \mathbb{K}$ & $\mathbb{K} C \ltimes(\widetilde{H}(n-2) \ltimes \Lambda(n-2))$ \\
\hline$F$ & $s(\mathfrak{g l}(1 \mid 1) \oplus \mathfrak{g l}(1 \mid 1)) /\left\langle I_{4}\right\rangle$ & $\mathfrak{s l} \ltimes \mathfrak{i s t r}\left(g l(1,1)_{+}\right)$ \\
\hline$J P(0, n-3)$ & $\widetilde{H}(n-2) \ltimes\left(\Lambda(n-2) /\left\langle\xi_{1} \cdots \xi_{n-2}\right\rangle\right)$ & $\mathbb{}$ \\
\hline$g l(1,1)_{+}$ &
\end{tabular}

Again, if $\mathfrak{s t r}(V)$ is isomorphic to $\mathfrak{i s t r}(V)$, we only wrote it once and the notation is explained in Appendix A. The action of $\mathfrak{s l}_{2}$ on $\operatorname{istr}\left(g l(1,1)_{+}\right)$is the adjoint action by using the embedding of $\mathfrak{s l}_{2}$ in $D(2,1 ;-1)$. The following isomorphisms exist in the list of Jordan superalgebras:

$$
(1,2)_{+} \cong D_{1}, \quad D_{t} \cong D_{t^{-1}} .
$$

Furthermore, also the simple Jordan superalgebras $J P(0,1)$ and $D_{-1}$ appear in the literature, but they are isomorphic to $g l(1,1)_{+}$, so they are already included in the table.

6.2. The non-unital finite dimensional simple Jordan superalgebra. The full list of finite dimensional simple Jordan superalgebras in $[\mathrm{Ka} 2, \mathrm{CK}]$ contains only one Jordan superalgebra which is non-unital. In [Ka2] it is denoted by $K$. The algebra $K$ is defined as

$$
K=\langle a\rangle \oplus\left\langle\xi_{1}, \xi_{2}\right\rangle, \quad|a|=\overline{0},\left|\xi_{1}\right|=\left|\xi_{2}\right|=\overline{1},
$$

with multiplication satisfying $a^{2}=a, a \xi_{i}=\frac{1}{2} \xi_{i}$ and $\xi_{1} \xi_{2}=a$.

A straightforward calculation implies

$$
\mathfrak{i s t r}(K)=\mathfrak{s t r}(K)=\operatorname{Inn}(K, K) \cong \mathfrak{s l}(1 \mid 2), \text { and } \operatorname{Der}(K, K) \cong \mathfrak{g l}(1 \mid 2) .
$$

This gives a counterexample to the statement in Proposition 3.7(1) for non-unital Jordan superalgebras. For $K$, the sums in Definitions 3.2 and 3.3 are direct.

One also finds

$$
\operatorname{Ko}(K) \cong \mathfrak{p s l}(2 \mid 2) .
$$

By construction, $\widetilde{\operatorname{Ko}}(K)$ is an extension over $\operatorname{Ko}(K)$. As $\mathfrak{i s t r}(K) \cong \operatorname{Inn}(K, K)$, it follows easily that the same is true for $\operatorname{Kan}(K)$. The algebras $\widetilde{\operatorname{Ko}}(K)$ and $\operatorname{Kan}(K)$ can hence be described in terms of $\operatorname{Out}(\operatorname{Ko}(K)) \cong \mathfrak{s l}_{2}$ :

- $\widetilde{\mathrm{Ko}}(K) \cong \mathfrak{p g l}(2 \mid 2)$ is the extension of $\mathbb{K}$ over $\mathrm{Ko}(K)$ corresponding to the morphism $\mathbb{K} \rightarrow \mathfrak{s l}_{2}$, where $1 \in \mathbb{K}$ is mapped to a semisimple element of $\mathfrak{s l}_{2}$.

- $\operatorname{Kan}(K)$ is the extension of $\mathbb{K}$ over $\operatorname{Ko}(K)$ corresponding to the morphism $\mathbb{K} \rightarrow \mathfrak{s l}_{2}$, where $1 \in \mathbb{K}$ is mapped to a nilpotent element of $\mathfrak{s l}_{2}$.

In particular we find that

$$
\widetilde{\operatorname{Ko}}(K) \neq \operatorname{Der}(\operatorname{Ko}(K)) \quad \text { and } \quad \operatorname{Kan}(K) \neq \operatorname{Ko}(K) .
$$


This gives counterexamples to the statements in Propositions 5.5 and 4.3, for non-unital Jordan superalgebras. By Remark 4.6 and the above, we do have

$$
\operatorname{Ti}\left(K, \operatorname{Inn}(K), \mathfrak{s l}_{2}\right) \cong \operatorname{Ti}\left(K, \operatorname{Der}(K), \mathfrak{s l}_{2}\right) \cong \operatorname{Ko}(K) \cong \mathfrak{p s l}(2 \mid 2) .
$$

For the 3-grading on $\mathfrak{p s l}(2 \mid 2)$ corresponding to the interpretation as $\operatorname{Ko}(K)$, the algebra $\mathfrak{g}=$ $\operatorname{Out}\left(\mathfrak{p s l}(2 \mid 2) \cong \mathfrak{s l}_{2}\right.$ is 3 -graded where $\mathfrak{g}_{i}$ has dimension one for $i \in\{-1,0,1\}$. This is in sharp contrast with Lemma 5.11 for the unital case. By Proposition 5.8, $\widetilde{\mathrm{Ko}}(K)$ is the subalgebra of $\operatorname{Der}(\operatorname{Ko}(K))$ where only the degree 0 derivations are added to $\operatorname{Ko}(K)$. In the same way, $\operatorname{Kan}(K)$ is the subalgebra of $\operatorname{Der}(\operatorname{Ko}(K))$ where only the degree 1 derivations are added to $\operatorname{Ko}(K)$.

\section{Appendix A. The Lie superalgebras of type $A, P, Q$ and $H$}

Consider an algebraically closed field $\mathbb{K}$ of characteristic zero. We quickly review the Lie superalgebras of type $A, P, Q$, and $H$, as different notations appear in literature. Our nomenclature is based on [CW]. See also [CW, Theorem 1.11] for the list of simple finite dimensional Lie superalgebras.

A.1. Type A. The general linear superalgebra $\mathfrak{g l}(m \mid n)$ is defined as $\operatorname{End}\left(\mathbb{K}^{m \mid n}\right)$, with multiplication given by the super commutator. Define the supertrace for a matrix $A \in \mathfrak{g l}(m \mid n)$ as $\operatorname{str}(A):=\sum_{i}(-1)^{|i|} A_{i i}$, where $|i|=\overline{0}$ for $i \leq m$ and $|i|=\overline{1}$ for $m+1 \leq i \leq m+n$. The special linear superalgebra is

$$
\mathfrak{s l}(m \mid n)=\{A \in \mathfrak{g l}(m \mid n) \mid \operatorname{str}(A)=0\},
$$

If $m \neq n$ then $\mathfrak{s l}(m \mid n)$ is simple. If $m=n$ then $\left\langle I_{2 n}\right\rangle$, with $I_{2 n}$ the identity matrix, is an ideal in $\mathfrak{s l}(n \mid n)$ and

$$
\mathfrak{p s l}(n \mid n):=\mathfrak{s l}(n \mid n) /\left\langle I_{2 n}\right\rangle
$$

is simple for $n>1$. Similarly, we set

$$
\mathfrak{p g l}(n \mid n):=\mathfrak{g l}(n \mid n) /\left\langle I_{2 n}\right\rangle .
$$

A.2. Type P. The periplectic Lie superalgebra is the subalgebra of $\mathfrak{g l}(n \mid n)$ defined as

$$
\mathfrak{p} \mathfrak{e}(n):=\left\{\left(\begin{array}{cc}
a & b \\
c & -a^{t}
\end{array}\right) \mid a, b, c \in \mathbb{K}^{n \times n} \text { with } b^{t}=b, c^{t}=-c\right\} .
$$

The special periplectic Lie superalgebra is defined as

$$
\mathfrak{s p e}(n):=\{x \in \mathfrak{p}(n) \mid \operatorname{tr}(a)=0\} .
$$

It is simple for $n \geq 3$.

A.3. Type Q. The queer Lie superalgebra is the subalgebra of $\mathfrak{g l}(n \mid n)$ defined as

$$
\mathfrak{q}(n):=\left\{\left(\begin{array}{cc}
a & b \\
b & a
\end{array}\right) \mid a, b \in \mathbb{K}^{n \times n}\right\} .
$$

Remark that $\operatorname{str}(X)=0$ for all $X \in \mathfrak{q}(n)$. The special queer Lie superalgebra is defined as

$$
\mathfrak{s q}(n):=\left\{\left(\begin{array}{cc}
a & b \\
b & a
\end{array}\right) \mid a, b \in \mathbb{K}^{n \times n}, \operatorname{tr}(b)=0\right\} .
$$

The projective special queer Lie superalgebra is defined as

$$
\mathfrak{p s q}(n):=\mathfrak{s q}(n) /\left\langle I_{2 n}\right\rangle .
$$


It is simple for $n \geq 3$. We also define the projective queer Lie superalgebra as

$$
\mathfrak{p q}(n):=\mathfrak{q}(n) /\left\langle I_{2 n}\right\rangle .
$$

A.4. Type H. Let $\Lambda(n)$ be the exterior algebra generated by $\xi_{1}, \ldots, \xi_{n}$. The indeterminates hence satisfy

$$
\xi_{i} \xi_{j}=-\xi_{j} \xi_{i}
$$

This is an associative superalgebra where the generators are odd, $\left|\xi_{i}\right|=\overline{1}$. We also consider a compatible $\mathbb{Z}$-grading, by setting $\operatorname{deg} \xi_{i}=1$. Denote by $W(n)$ the algebra of derivations of the associative superalgebra $\Lambda(n)$. The Lie superalgebra $W(n)$ is simple for $n \geq 2$.

On $\Lambda(n)$, we define the following Poisson superbracket

$$
\{f, g\}:=(-1)^{|f|}\left(\sum_{i=1}^{n-2} \partial_{\xi_{i}} f \partial_{\xi_{i}} g+\partial_{\xi_{n-1}} f \partial_{\xi_{n}} g+\partial_{\xi_{n}} f \partial_{\xi_{n-1}} g\right),
$$

for $f$ and $g$ in $\Lambda(n)$. Then $(\Lambda(n),\{\cdot, \cdot\})$ becomes a Lie superalgebra with ideal $\langle 1\rangle$. Consider the following Lie superalgebras

$$
\widetilde{H}(n):=\Lambda(n) /\langle 1\rangle \text { and } H(n):=[\widetilde{H}(n), \widetilde{H}(n)] .
$$

Note that $\widetilde{H}(n)=H(n) \oplus \mathbb{K} \xi_{1} \cdots \xi_{n}$ as super vector spaces. The Lie superalgebra $H(n)$ is simple for $n \geq 4$. We can embed $H(n)$ and $\widetilde{H}(n)$ into $W(n)$, using $f \mapsto\{f, \cdot\}$.

Consider $C:=\sum_{i=1}^{n} \xi_{i} \partial_{\xi_{i}} \in W(n)$, then $\mathbb{K} C \ltimes \widetilde{H}(n)$ is naturally defined as a subalgebra of $W(n)$.

We also define the semidirect product $\widetilde{H}(n-2) \ltimes \Lambda(n-2)$, where the action of $\widetilde{H}(n-2)$ on $\Lambda(n-2)$ is given by the Poisson superbracket on $\Lambda(n-2)$, while the bracket of $\Lambda(n-2)$ is trivial. We further introduce, $\mathbb{K} C \ltimes(\widetilde{H}(n-2) \ltimes \Lambda(n-2))$, where $C$ acts by $[C, f]=(\operatorname{deg} f-2) f$ for $f \in \widetilde{H}(n-2)$ and by $[C, g]=\operatorname{deg} g$ for $g \in \Lambda(n-2)$.

Acknowledgment. SB is a PhD Fellow of the Research Foundation - Flanders (FWO). KC is supported by Australian Research Council Discover-Project Grant DP140103239 and a postdoctoral fellowship of the Research Foundation - Flanders (FWO).

The authors thank Hendrik De Bie, Tom De Medts and Erhard Neher for helpful discussions and comments.

\section{REFERENCES}

[AMR] D. Alekseevsky, P. Michor, W. Ruppert. Extensions of super Lie algebras. J. Lie Theory 15 (2005), no. $1,125-134$.

[BDS] L. Boelaert, T. De Medts, A. Stavrova. Moufang sets and structurable division algebras. Preprint: ArXiv:1603.00780.

[CK] N. Cantarini, V. G. Kac. Classification of linearly compact simple Jordan and generalized Poisson superalgebras. J. Algebra 313 (2007), no. 1, 100-124.

[CW] S.J. Cheng, W. Wang. Dualities and representations of Lie superalgebras. Graduate Studies in Mathematics, 144. American Mathematical Society, Providence, RI, 2012.

[FK] J. Faraut, A. Korányi. Analysis on symmetric cones. Oxford Mathematical Monographs. Oxford Science Publications. The Clarendon Press, Oxford University Press, New York, (1994)

[GN] E. García, E. Neher. Tits-Kantor-Koecher superalgebras of Jordan superpairs covered by grids. Comm. Algebra 31 (2003), no. 7, 3335-3375. 
[Ja] N. Jacobson. Structure groups and Lie algebras of Jordan algebras of symmetric elements of associative algebras with involution. Advances in Math. 20 (1976), no. 2, 106-150.

[Ka1] V. G. Kac. Lie superalgebras. Advances in Math. 26 (1977), no. 1, 8-96.

[Ka2] V. G. Kac. Classification of simple Z-graded Lie superalgebras and simple Jordan superalgebras. Comm. Algebra 5 (1977), no. 13, 1375-1400.

[KMZ] V. G. Kac, C. Martinez, E. Zelmanov. Graded simple Jordan superalgebras of growth one. Mem. Amer. Math. Soc. 150 (2001), no. 711.

[Kan] I. L. Kantor. Transitive differential groups and invariant connections in homogeneous spaces. Trudy Sem. Vektor. Tenzor. Anal. 13 (1966) 310-398.

[Kan2] I. L. Kantor. Jordan and Lie superalgebras determined by a Poisson algebra. Amer. Math. Soc. Transl. Ser. 2, 151, Amer. Math. Soc., Providence, RI, 1992.

$[\mathrm{KM}] \quad$ I. Kashuba, M. E. Martin. The variety of three-dimensional real Jordan algebras. J. Algebra Appl. 15 (2016), no. 8, 1650158

[KS] I. Kashuba, V. Serganova. On the Tits-Kantor-Koecher construction of unital Jordan bimodules. Preprint: ArXiv:1502.07407.

[Ko] M. Koecher. Imbedding of Jordan algebras into Lie algebras. I. Amer. J. Math. 89 (1967) 787-816.

[Kr] S. V. Krutelevich. Simple Jordan superpairs. Comm. Algebra 25 (1997), no. 8, 2635-2657.

[Lo] O. Loos. Jordan pairs. Lecture Notes in Mathematics, Vol. 460. Springer-Verlag, Berlin-New York, 1975.

[MZ] C. Martinez, E. Zelmanov. Representation theory of Jordan superalgebras I. Trans. AMS, 362, (2010), no. $2,815-846$.

[McC] K. McCrimmon. A taste of Jordan algebras. Universitext, Springer-Verlag, New York, 2004.

[Sc] M. Scheunert. The theory of Lie superalgebras. An introduction. Lecture Notes in Mathematics, 716. Springer, Berlin, 1979.

[Sh] A. S. Shtern. Representations of finite dimensional Jordan superalgebras of Poisson bracket. Comm. Algebra, 23, (1995), no. 5, 1815-1823.

[Sp] T. Springer. Jordan algebras and algebraic groups. Ergebnisse der Mathematik und ihrer Grenzgebiete, Band 75. Springer-Verlag, New York-Heidelberg, 1973

[Ti] J. Tits. Une classe d'algèbres de Lie en relation avec les algèbres de Jordan. Nederl. Akad. Wetensch. Proc. Ser. A 65 = Indag. Math. 24 (1962) 530-535.

SB: Department of Mathematical Analysis, Faculty of Engineering and Architecture, Ghent University, Krijgslaan 281, 9000 Gent, Belgium; E-mail: Sigiswald.Barbier@UGent.be

KC: School of Mathematics and Statistics, University of Sydney, NSW 2006, Australia; E-mail: kevin. coulembier@sydney. edu. au 